rates among HIV-negative and HIV-unknown MSM, combined, increased 64\% (83.0 to 136.5 per 100,000$)$, while rates among MSM with diagnosed HIV increased 17\% during 2011-2014 (1,061.5 to $1,237.3$ per 100,000$)$ before decreasing $6 \%$ to $1,229.5$ in 2015 . Rate ratios comparing reported P\&S syphilis rates among MSM living with diagnosed HIV to HIV-negative and HIV-unknown MSM decreased annually during this period from 12.8 to 9.0 .

Conclusion During the most recent five-year period for which data are available, rates of reported P\&S syphilis increased among MSM diagnosed with HIV, as well as MSM not diagnosed with HIV. Although rates are higher among MSM diagnosed with HIV, larger relative increases in rates among MSM not diagnosed with HIV and subsequent declining rate ratios indicated that differences between MSM with diagnosed HIV and HIV-negative or HIV-unknown MSM diminished over time.

Disclosure No significant relationships.

\section{P529 EARLY SEXUAL DEBUT AND NON-CONSENSUAL SEX AMONG CHINESE MEN WHO HAVE SEX WITH MEN: A MULTI-CITY CROSS-SECTIONAL STUDY}

${ }^{1}$ Weiming Tang*, ${ }^{1}$ Yehua Wang, ${ }^{1}$ Jason Ong, ${ }^{1}$ Dan Wu, ${ }^{2} \mathrm{M}$ Kumi Smith, ${ }^{3} \mathrm{H}$ ongyun $\mathrm{Fu}$ ${ }^{1}$ Wenting Huang, ${ }^{4}$ Chongyi Wei, ${ }^{1}$ Joseph Tucker. ${ }^{1}$ UNC Project-China, Guangzhou, China; ${ }^{2}$ University of Minnesota Twin Cities, Epidemiology and Community Health, Minneapolis, USA; ${ }^{3}$ Eastern Virginia Medical School, Norfolk, USA; ${ }^{4}$ Rutgers, USA

\subsection{6/sextrans-2019-sti.607}

Background Early sexual debut and non-consensual sex among men who have sex with men (MSM) may contribute to adult high-risk behaviors and STI transmission. Most knowledge about early MSM sex comes from high-income countries. This study examined the prevalence and factors associated with the early sexual debut and non-consensual sex at sexual debut among Chinese MSM.

Methods An online cross-sectional study was conducted among MSM who were born as a male, $\geq 16$ years-old, and ever engaged in anal sex with a man in China in 2016. Participants answered questions regarding sociodemographics, condomless sex, and HIV testing history. Early sexual debut was defined as having anal sex before 18 . Non-consensual sex was defined as "where a person do sexual things to you that you did not want them to do." Multivariable logistic regression was used to identify factors associated with the early sexual debut and non-consensual sex.

Results Overall, 2105 men completed the survey. Among them, $85.9 \%$ were never married, and $35.4 \%$ had high school or less education. The mean age of sexual debut was 20.82 $(S D=5.30)$ years. About one-fifth $(20.1 \%, 424 / 2105)$ of men experienced early sexual debut, and 4.9\% (104/2105) experience non-consensual sex at sexual debut. Results from multivariable logistic regression models showed that early sexual debut was associated with having more male partners in the last three months (adjusted OR 1.08 [95\% CI 1.04-1.13]) and condomless sex in the last three months (1.49 [1.18-1.89]). MSM whose sexual debut was non-consensual were more likely to have tested for HIV (1.56 [1.01-2.42]), have had recent condomless sex (1.70 [1.13-2.56]), and to experience early sexual debut (2.54 [1.65-3.89]).

Conclusion Many Chinese MSM experience early sexual debut and non-consensual sex at sexual debut. Given the limited coverage of sexual health education in China, this has implications for expanding education and research about sexuality among youth.

Disclosure No significant relationships.

\section{P530 ADDRESSING UNDERSERVED MEN WHO HAVE SEX WITH MEN (MSM): ADVANCING THE SEXUAL HEALTH APPROACH FOR MSM IN VANCOUVER, CANADA}

${ }^{1}$ Tessa Lawson Tattersall, ${ }^{1}$ Nathan Lachowsky*, ${ }^{2}$ Mark Hull. ${ }^{1}$ University of Victoria, School of Public Health and Social Policy, Victoria, Canada; ${ }^{2}$ British Columbia Centre for Excellence in HIVIAIDS, Vancouver, Canada

\subsection{6/sextrans-2019-sti.608}

Background Men who have sex with men (MSM) are disproportionately overrepresented in the resurgence of sexually transmitted and blood-borne infections (STBBI) indicating a need to understand how access to sexual health services can be adapted for the improvement of the health and well-being of MSM. Our objective was to contextualize the access to and provision of MSM sexual health care with a syndemic lens. Accordingly, this study employs qualitative understanding of access to sexual health care, with particular focus on preexposure prophylaxis, for MSM in Vancouver.

Methods We conducted five focus groups from 12/2016-07/ 2017 with sexual health service providers serving MSM and members from the MSM community who have accessed sexual health care. Focus groups were audio recorded and transcribed verbatim. We iteratively analyzed data through a constant comparative technique to identify the accessibility of sexual health care for MSM in Vancouver with inference to syndemic effect. Results We identified service provider and MSM community attitudes and the structural concerns impacting access to sexual health care for MSM in Vancouver, Canada. Access to sexual health care assembled into three themes: (1) Perceptions and categorization of underserved; (2) Value of specialized MSM sexual health service; and (3) Capacity: reaching and referring. The first represents syndemics, reaching undiagnosed, representation and intersectionality, and specialized service providers as encouraging PrEP gatekeepers. The second combines MSM friendly environment, separation of sexual from other health care, and fear of association: community stigma and internalized shame. The third includes service promotion, capacity for HIV care, lack of counseling, and referral adequacy.

Conclusion MSM experience many biosocial interactions that negatively impact their access to sexual health care. Therefore, sexual health care would be more likely accessed if conditions were addressed together rather than separately. Findings offer social and structural-level interventions to address stigma and syndemic influence within MSM sexual health care delivery. Disclosure No significant relationships. 